\title{
Human Amniotic Mesenchymal Stem Cells Inhibit aGVHD in Humanized NPG Mice by Regulating Balance of Treg Versus T Effector Cells
}

\section{Ya Gao}

Southern Medical University Nanfang Hospital

\section{Weiru Li}

Southern Medical University Nanfang Hospital

\section{Xiaoyin Bu}

Southern Medical University Nanfang Hospital

\section{Ying Xu}

Southern Medical University Nanfang Hospital

\section{Shengchun Cai}

Southern Medical University Nanfang Hospital

\section{Jinman Zhong}

Southern Medical University Nanfang Hospital

\section{Meixue Du}

Southern Medical University Nanfang Hospital

\section{Haitao Sun}

Southern Medical University Zhujiang Hospital

\section{Liping Huang}

Southern Medical University Nanfang Hospital

\section{Yongjian He}

Southern Medical University Nanfang Hospital

\section{Xiumei Hu}

Southern Medical University Nanfang Hospital

\section{Qifa Liu}

Southern Medical University Nanfang Hospital

Hua Jin

Southern Medical University Nanfang Hospital

\section{Qian Wang}

Southern Medical University Zhujiang Hospital

\section{Baohong Ping ( $\nabla$ nfyypingbaohong66@163.com )}

Nanfang Hospital https://orcid.org/0000-0003-1177-5904 
Research

Keywords: Acute graft versus host disease, Humanized mouse model, amniotic mesenchymal stem cells, balance of Treg vs. T effector cells

Posted Date: January 16th, 2021

DOl: https://doi.org/10.21203/rs.3.rs-142719/v1

License: (c) (i) This work is licensed under a Creative Commons Attribution 4.0 International License.

Read Full License 


\section{Abstract}

Background: Acute graft versus host disease(aGVHD) occurs when immunocompetent T cells in the donated tissue recognize the recipient as foreign after allo-HSCT, which can severely affect quality of life. Although previous studies indicated that MSC may be a salvage therapeutic agent for aGVHD, the mechanism is not yet fully clear. The aim of this study was to explore the therapeutic efficacy and underlying mechanisms of hAMSCs transplantation for humanized aGVHD mouse model.

Methods: We established a novel xenogeneic (xeno)-aGVHD humanized mouse models by transplanting purified human T cells from peripheral blood into immunodeficient NOD-Prkdc ${ }^{\text {scid }} \mid 2$ ry ${ }^{\text {null }}$ (NPG) mouse. In those mouse models, lymphocyte infiltration of target organs was detected by HE staining method and immunohistochemistry respectively. The biological characteristics of hAMSCs were investigated. hAMSCs labeled with GFP were administered to NPG mice to explore the homing ability of hAMSCs. Mice are divided into normal(control) group, aGVHD group and hAMSCs treatment group. After 3 days of injection of PBMC, hAMSCs and PBS were given into the different groups. T effector and Treg cells levels of each group in target organs were detected by flow cytometry and cytometric bead array (CBA).

Results: We successfully established xenogeneic aGVHD "humanized model" by using NODPrkdc ${ }^{\text {scid }} \mid 2$ ry ${ }^{\text {null }}$ (NPG) mice, which showed lymphocytes infiltration in the liver, spleen and lung. hAMSCs therapy improved systemic inflammation and inhibited aGVHD in humanized mouse through reduced villous blunting and lymphocyte infiltration into the lamina propria of the gut while reducing vascular endothelialitis and lymphocyte infiltration into the parenchyma of the liver and lung. In addition, hAMSCs suppresses xenogenesis $C D 3+/ C D 4+$ and $C D 3+/ C D 8+T$ cell concentration and increases the proportion of Treg cells. Our data also show that hAMSC can reduce the level of IL-17A, INF- $y$, TNF and IL-2 that involved in the pathogenesis of aGVHD target organs.

Conclusions: NPG murine environment is capable of activating human T cells to produce aGVHD pathology to mimic aGVHD in humans. hAMSCs controlled aGVHD by decreasing inflammatory cytokine secretion within target organs through modulating balance of Treg versus $T$ effector cells in humanized mice.

\section{Introduction}

Graft-versus-host disease (GVHD) is reported as a syndrome in which donor immunocompetent cells recognize and attack host tissues in immunocompromised allogeneic recipients after hematopoietic stem cell transplantation (HSCT) [1, 2]. Steroids are the appropriate first line treatment for this disease, however, there are many treatment-related complications [3]. Besides, around half of patients develop aGVHD, even when preventive measures are adequately used [4]. Thus, there is an urgent need to develop more potent immunosuppressive treatment strategies for patients suffering from acute steroid refractory aGVHD while maintaining the graft versus tumor effect to avoid a potential rise in relapse-related mortality. 
Mesenchymal stem cells (MSCs) are non-hematopoietic adult multipotent progenitor cells that can be isolated from various adult tissues. Therapeutic infusion of MSCs is now a leading strategy for the treatment of aGVHD in clinical [5]. Human amniotic mesenchymal stem cells (hAMSCs) are a sort of MSC which can be easily and safely isolated from amniotic membrane of health pregnant women (medical waste) and have been recognized as one of the most promising stem cells in the field of regenerative medicine [6]. Well-growing hAMSCs express CD90, CD105, CD73 but not hematopoietic cell markers such as CD45, CD34, HLA-DR and CD11b. hAMSCs possess immunomodulatory properties that are thought to enable damaged tissues to form a balanced inflammatory and regenerative micro-environment in the presence of vigorous inflammation generally [7]. Furthermore, immunomodulatory activity of hAMSCs is equal to or higher than that of human bone marrow MSCs [8]. Thus, the active proliferative potential, low immunogenic profile, anti-inflammatory function of hAMSCs can be beneficial to the therapy of inflammation-related diseases, and immunosuppression. Kenichi Yamahara et al [9] found that patients with steroid-refractory aGVHD infusion of hAMSCs is safety and efficacy. Our previously study demonstrated that hAMSCs can induce Th1 cells into Th2 cells in vitro [10]. However, reports about the mechanisms by which hAMSCs exert their therapeutic effects in vivo are rare.

Due to ethical constraints, relevant research cannot be conducted on humans, GVHD pathogenesis has been mostly studied in mouse-to-mouse models of transplantation. Until recently, C57/BI6 (H2b) donors into BALB/c ( $\mathrm{H} 2 \mathrm{~d})$ recipients has been widely used in the establishment of aGVHD model mice. However, mouse to mouse aGVHD model still has some limitations. To generate a system whereby human T-cellmediated aGVHD can be studied and manipulated in vivo, xenogeneic transplant models have been rapidly developed [11]. Currently, the most popular immune deficient mouse strains, NOD/SCID-IL2RY ${ }^{-1 /}$,

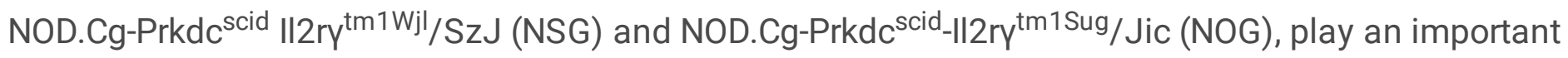
role in study of immunological pathologies mechanism of GVHD and the improvement of therapy. Those mice lack T, B and NK cells, and also have reduced function of macrophages and dendritic cells that make human peripheral blood mononuclear cells (PBMCs) engraftment well. Transplantation of PBMCs can cause an aGVHD syndrome because of human APCs process mouse antigens and present them in the presence of class II MHC [12]. Compared to mouse models of aGVHD, the "humanized model" has many advantages such as the use of human cells to induce and control xenogeneic (xeno)-aGVHD, the possibility of using donors with a high genetic diversity, and the possibility of using older donors previously exposed to various pathogens [13]. Here, we used NOD-Prkdcscidll2rynull (NPG) mice to establish xenogeneic aGVHD "humanized model" and investigated the therapeutic potential of hAMSCs in preventing aGVHD in vivo. We demonstrate that hAMSCs can ameliorate aGVHD in an allospecific manner via addition of Tregs cell and reduction of inflammatory cytokine secretion in target organs. Our results support further application of human amniotic mesenchymal stem cells to prevent and treat aGVHD in clinical.

\section{Methods}

\section{Isolation and culture of cells}


Human placentas were obtained from caesarean sections from healthy women with informed consent. The amnion layer was separated from chorion layer and washed several times with PBS and cut into pieces of $1.0 \mathrm{~cm}^{2}$ and digested with $0.25 \%$ trypsin at $37^{\circ} \mathrm{C}$ for $30 \mathrm{~min}$. After that, amniotic epithelial cells were removed gently. And then, the amnion was digested again under the same condition. Finally, the amnion was washed by PBS and cut into $1 \mathrm{~mm}^{2}$ and cultured in a-MEM (1x; Gibco, USA, C12571) supplemented with 10\% FBS (Gibco, Brazil, A31608-02). All the hAMSCs used in experiments were thirdpassage to fifth-passage. Suspension of P3 hAMSCs were counted and adjusted into $1 \times 10^{6} \mathrm{cells} / \mathrm{mL}$.

\section{Identification of human amniotic mesenchymal stem cells}

For cell identification, cells were incubated with mouse anti-human CD90-PE antibody (BD Pharmingen ${ }^{\mathrm{TM}}$, catalog number: 561970), mouse anti-human CD105-PE antibody (eBioscience, catalog number: 12-105742), mouse anti-human CD73-PE antibody (BD Pharmingen ${ }^{\text {TM }}$, catalog number: 561014), mouse antihuman CD45 -PE antibody (BD Pharmingen ${ }^{T M}$, catalog number: 560975), mouse anti-human CD11b-PE$\mathrm{Cy}^{\mathrm{TM}}$ 7antibody (BD Pharmingen ${ }^{\mathrm{TM}}$, catalog number: 561098), mouse anti-human CD34-PE antibody (BD Pharmingen ${ }^{\mathrm{T}}{ }^{\mathrm{M}}$, catalog number: 560941) mouse and anti-human HLA-DR-PE antibody (BD Pharmingen ${ }^{\mathrm{TM}}$, catalog number: 560943 ) at $4^{\circ} \mathrm{C}$ for $30 \mathrm{~min}$. Cells were washed by PBS and adjusted into $400 \mu \mathrm{L}$ and then analyzed by flow cytometry.

\section{Adipogenic and osteogenic differentiation}

To analyze adipogenic and osteogenic differentiation, passage 3 hAMSCs were seeded at a density of $1.5 \times 10^{5}$ cells/well in a six-well plate. When the cells reached $100 \%$ confluence, cells were incubated in human mesenchymal stem cell adipogenic differentiation medium (Pythonbio, catalog number:

20191008), for 21 days, the adipogenic induction medium was replaced every 3 days. Oil red 0 (Solarbio, catalog number: G1260) staining was performed to assess the differentiation potential. For osteogenic differentiation, hAMSCs were cultured with human mesenchymal stem cell osteogenic differentiation medium (Pythonbio, catalog number: 20191009) for 21 days, induction medium was replaced every 3 days. The differentiation potential for osteogenesis was assessed by Alizarin Red (pH 4.2, $40 \mathrm{mM}$ ) (Solarbio, catalog number: G1452) staining.

\section{Animals}

NPG mice 8-10 weeks of age were obtained from Beijing Vitalstar Biotechnology Co.,Ltd (laboratory animal production license No.SCXK2016-0167). Animals were housed in a specific pathogen-free facility in microisolator cages, given autoclaved food and maintained on acidified autoclaved water and a solution of gentamicin. All animal experiments were approved by the laboratory animal ethics review committee of southern medical university. All animal procedures were in accordance with the National Institute about laboratory animal care and use.

\section{Human peripheral blood mononuclear cells collection}


Human PBMC were collected from healthy volunteers. Each donor singed written informed consent. PBMC were collected in sodium citrate and purified by Ficoll-Hypaque (Solarbio) density centrifugation and washed in phosphate-buffered saline (PBS). Then suspended in red blood cell lysis buffer (Solarbio) at $4^{\circ} \mathrm{C}$ for $15 \mathrm{~min}$ and washed again in PBS. And finally suspended in PBS for injection into NPG mice from tail vein.

\section{Induction of xenogeneic aGVHD in NPG mice}

All mice were given 200 cGy irradiation 3-4h before cell injection unless indicated otherwise. Then were injected with $3 \times 10^{6}$ of PBMC suspended into $500 \mu \mathrm{L}$ PBS intravenously. During all experiments, each mouse was graded according xeno-aGVHD clinical scoring system (Table 1). The symptoms of xenoGVHD including weight loss, hunched posture, ruffled fur, reduced mobility and diarrhea. Mice survived till the 28 day endpoint and those which suffered from severe xeno-GVHD (weight loss $₫ 25 \%$, severe hunched posture, severe ruffled fur, less or no mobility or hematochezia) were euthanized.

\section{GFP-labelled hAMSCs and tracing hAMSC in vivo}

hAMSCs were planted to 24 -well plate, cell concentration was $3 \times 10^{5} / \mathrm{mL}, 500 \mu \mathrm{L}$ per well. Then the $24-w e l l$ plate was placed in an incubator condition of $37^{\circ} \mathrm{C}$ and $5 \% \mathrm{CO}_{2} .24$ hours later, culture media was disposed, $250 \mu \mathrm{L}$ culture media and 1.6 $\mathrm{L}$ GFP-pseudovirion (Hanbio) were added, the rest of the $250 \mu \mathrm{L}$ culture media was added 4 hours later and then placed in the incubator. After 24 hours, culture media was disposed, $500 \mu \mathrm{L}$ culture media was added and then placed in the incubator. GFP labeled hAMSCs could be found by fluorescence microscope in two to three days. The suspension of GFP labeled hAMSCs was adjusted into $5 \times 10^{5}$ cells/mL and injected into NPG mice via tail vein. These mice were euthanized after $24 \mathrm{~h}$ and $72 \mathrm{~h}$ respectively, blood and tissues such as liver, spleen, lung and gut were collected. Single cell suspensions were obtained by grinding liver, spleen, lung, gut. Finally, single cell suspensions of tissues and blood were analyzed by flow cytometry to detected GFP labeled signal. For immunofluorescence, target organs were fixed with 4\% paraformaldehyde for 24 hours and then dehydrated with $30 \%$ sucrose. After more than 48 hours target organs were embedded in a frozen slicer at $-25^{\circ} \mathrm{C}$ and made into frozen slices. Recipient cells were distinguished by DAPI counterstaining.

\section{Histopathological analyses}

Tissues were collected at the time of necropsy, fixed in $10 \%$ buffered formalin, and embedded in paraffin. For routine histology, sections were stained with hematoxylin and eosin. For immunohistochemistry, sections were heated at $60^{\circ} \mathrm{C}$ for $20 \mathrm{~min}$, then deparaffinized and hydrated with xylene and alcohol baths for staining. The slides were heated in $0.01 \mathrm{M}$ citrate-buffer solution ( $\mathrm{pH} 6.0$ ) for 10 min in a microwave oven, then cooled down to room temperature. Immunohistochemical staining was performed with mAbs specific for human CD45 (Abcam, Massachusetts, US). For immunofluorescence, target organs were fixed with $4 \%$ paraformaldehyde for 24 hours and then dehydrated with $30 \%$ sucrose. After more than 48 hours target organs were embedded in a frozen slicer at $-25^{\circ} \mathrm{C}$ and made into frozen slices. Recipient cells were distinguished by DAPI counterstaining. 


\section{Flow Cytometric Analysis}

Mouse anti-human CD3-PE-Cyanine7 antibody (eBioscience, catalog number: 25-0038-42), mouse antihuman CD4-FITC antibody (BD Pharmingen ${ }^{\text {TM }}$, Catalog Number:340133), mouse anti-human CD8-PE (BD Pharmingen ${ }^{\mathrm{TM}}$, Catalog Number:340046), mouse anti-human CD25-APC antibody (Biolegend, Catalog Number: 302610), mouse anti-human Foxp3-PE(eBioscience Catalog Number: 12-4777-42). Peripheral blood, livers, spleens, lungs and guts were collected at the time of necropsy and analyzed by flow cytometry. Single cell suspensions were obtained by grinding liver, spleen, lung, gut and blood sample was processed with red blood cell lysis buffer (Solarbio) at $4^{\circ} \mathrm{C}$ according to the protocol. All samples were stained with antibodies or isotype matched control IgG for $30 \mathrm{~min}$ at $4^{\circ} \mathrm{C}$ in the dark. Then analyzed on a BD FACS Cantoll with FlowJo 10.0.

\section{Analysis of the cytokines by cytometric bead array (CBA)}

Cytometric bead array (CBA) human Th1/Th2/Th17 cytokine kit (BD, Catalog Number: 560484).

Peripheral blood and single cell suspensions of each target organ were centrifugate in a condition of $4^{\circ} \mathrm{C}$, $3000 \mathrm{rpm} / \mathrm{min}$ and $20 \mathrm{~min}$. Mixing human Th1/Th2/Th17 cytokine capture beads, vortex the mixed Capture Beads and add $50 \mu \mathrm{L}$ to all assay tubes, add $50 \mu \mathrm{L}$ of each sample to the appropriately labeled sample tubes and add $50 \mu \mathrm{L}$ of the Human Th1/Th2/Th17 PE detection reagent to all assay tubes. Incubate the assay tubes for 3 hours at room temperature, protected from light. add $1 \mathrm{~mL}$ of Wash Buffer to each assay tube and centrifuge at $200 \mathrm{~g}$ for 5 minutes, carefully aspirate and discard the supernatant from each assay tube. add $300 \mu \mathrm{L}$ of Wash Buffer to each assay tube to resuspend the bead pellet. Acquire the samples on the flow cytometer (BD FACS cantoll), analyze Human Th1/Th2/Th17 Cytokine data using FCAP Array software.

\section{Statistical analysis}

Comparisons between two means used the independent samples t-test. Comparisons of three or more means used one-way ANOVA. Survival curves were made with the Kaplan-Meier method. Pख0.05 were considered as significant, and for all figures, ${ }^{*} p<0.05,{ }^{* \star} p<0.01,{ }^{\star * \star} p<0.001$.

All statistical analyses were performed with GraphPad Prism 8.0.

\section{Results}

\section{A human xenogeneic acute GVHD model was successfully established in NOD-Prkdc ${ }^{\text {scid }} \| 2 r^{\text {null }}(\mathrm{NPG})$ mice}

To mimic GVHD in humans, we established a xenogeneic (xeno)-aGVHD humanized mouse by using NPG mice. First, to figure out whether low-dose irradiation is necessary for establishing xeno-aGVHD model, three groups of NPG mice were set up to receive PBS only, PBMC only or PBMC following 200 cGyirradiation [Fig 1A]. NPG mice received 200 cGy irradiation before human PBMC injected from tail vein 
with $3 \times 10^{6}$ all developed symptoms of xeno-aGVHD, which can mimic human aGVHD process, including weight loss, hunched posture, ruffed fur, reduced mobility, damaged skin, diarrhea and hematochezia compared with those mice implanted PBMC only. NPG mice received 200 cGy irradiation before $3 \times 10^{6}$ human PBMC injected from tail vein died within 12 to 14 days after cell transfer. In contrast, those NPG mice only received human PBMC survived for 28 days, suggesting that no significant xenogeneic response was involved in this model [Fig 1B, C]. Similar to humans, NPG mouse with aGVHD showed severe inflammation, leukocyte infiltration, necrosis, and tissue damage in target organs such as lung, liver and spleen [Fig 1D]. Lump-like PBMC infiltration were found in liver, spleen and lung [Fig 1E]. These results indicated that the aGVHD induced by xenogeneic donor hPBMCs in NPG mice was mediated mainly by human T cells. PBMC in blood were detected by flow cytometry, which confirmed that PBMC were injected into body [Fig 1E].

\section{Identification of hAMSCs in vitro and GFP labelled hAMSCs tracer cells in vivo}

Passage 3 hAMSCs appeared to be spindle- or polygon-shaped in morphology and reached $70-80 \%$ fusion after $48 \mathrm{~h}$ in culture [Fig 2A]. hAMSCs possessed adipogenic and osteogenic differentiation potentials in vitro [Fig $2 \mathrm{~B}, \mathrm{C}$ ]. Flow cytometry analysis of hAMSCs surface antigen phenotype revealed that CD90, CD105, and CD73 were expressed, while CD45, CD34, HLA-DR and CD11b were not. [Fig 2D]. As GFP gene was present in GFP-pseudovirion, the hAMSCs transduced with pseudovirion produced high level of GFP under fluorescence microscopy [Fig 2E]. After 24 and 72 hours hAMSCs-GFP injected into NPG mice, experimental mice were euthanized. hAMSCs-GFP could be detected in liver, spleen, lung and

gut after 24 hours. However, few GFP labeled cells could be found in target organs except lung after 72 hours [Fig 3A]. Flow cytometry showed the same results. The frequency of hAMSCs-GFP after 72 hours was higher than that after 24 hours in lung, whereas the proportion of GFP labeled cells after 72 hours was lower in liver, spleen and gut [Fig 3B]. These results demonstrated that after intravenous injection, hAMSCs-GFP can migrate to the damaged target organs.

\section{hAMSCs alleviated the clinical symptoms and improved overall survival in murine model of aGVHD}

In order to verify the immunosuppressed ability of hAMSCs in vivo, we tested hAMSCs in the xeno-aGVHD model [Fig 4A]. hAMSCs were collected from healthy maternal placenta that used for establishing the humanized recipient mice. As shown in [Fig 4B], hAMSCs can significantly ameliorated the severity of acute GVHD in terms of weight loss and disease score. Moreover, xeno-aGVHD model injected with hAMSCs survived for 26 to 28 days, in contrast, xeno-aGVHD model received PBS died within 6 to 18 days after transplantation. hAMSCs significantly ameliorated the severity of acute GVHD in terms of weight loss and disease score within 28 days of transplantation. Moreover, hAMSCs prevented leukocyte infiltration and reduced pathology in the lung, liver, gut and spleen on day 6 after transplantation [Fig 4C]. These results demonstrated that hAMSCs control aGVHD clinical symptoms in xeno-aGVHD mouse.

\section{hAMSCs modulate the balance of Treg versus T effector cells in humanized mice.}


To investigate the effect of hAMSCs on T effector cells and Treg cells, target organs of mice were grinding and analyzed by flow cytometry. We found that the proportion of $\mathrm{CD} 3+/ \mathrm{CD} 4^{+} \mathrm{T}$ cells of liver, spleen, lung and gut in mice receiving hAMSCs treatment were significantly lower than those untreated mice [Fig 5A]. Consistent with CD3+/CD4+T cells, the proportion of CD3+/CD8 ${ }^{+} \mathrm{T}$ cells in target organs of mice injected hAMSCs was also lower compared with untreated mice [Fig 5B]. We further found that CD4+CD25+FOXP3+Tregs from all target organs of xeno-aGVHD mice injected with hAMSCs were increased, especially in gut and peripheral blood [Fig 5C]. Our data support the finding that hAMSCs treatment significantly inhibited the proportion of donor hCD3+/CD $4+\mathrm{T}$ and $\mathrm{hCD} 3+/ \mathrm{CD} 8+\mathrm{T}$ cells and increased the proportion of Treg cells in target organs in vivo from day 3 to day 6 after transplantation.

\section{hAMSCs control aGVHD by decreasing inflammatory cytokine secretion in target organs}

hAMSCs treatment significantly inhibited the secretion of human inflammatory cytokines, like IL-17A, IFN$Y$, TNF and IL-2 from the lung, liver, gut or blood on day 3 after transplantation. [Fig 6A-D]. Especially in gut, which is the important aGVHD target organ, we found that IL-2, IFN- $\gamma$, IL-17A were suppressed by hAMSCs significantly. These results demonstrated that hAMSCs ameliorate human allogeneic acute GVHD by reduction of inflammatory cytokines secretion in target organs.

\section{Discussion}

Numerous reports have shown that mesenchymal stem cells (MSCs) yield a favorable therapeutic benefit for GVHD and most clinical trials have used MSCs [5, 14], nevertheless, the controversy about their safety and efficacy still remains. Although, BM is the main source of MSCs, the use of BM-MSCs is not always acceptable because of the invasive harvesting procedure, besides, the number of BMSCs declines with increasing age $[15,16]$. Placenta tissue-derived MSCs have the advantage of being readily available and easy to collect from a waste product, which has been reported to contain a population of multipotent stem cells exhibiting characteristics of MSCs [17]. Therefore, MSC from amniotic membrane might be a better option than BM-MSCs. However, it is rarely reported that human amniotic mesenchymal stem cells (hAMSCs) can reduce GVHD in vivo and its mechanism is unowning this current study, we establish a human allogeneic acute GVHD model in "humanized mice" by adoptive transfer of allogeneic hPBMCs into NOD-Prkdc ${ }^{\text {scid }} \| 2 r^{\text {null }}$ (NPG) mice that with an immunodeficiency system $[18,19]$. The humanized aGVHD model is mediated mainly by donor $T$ cells and characterized by disease appearance (hunching, activity, ruffling, and diarrhea), recruitment of alloreactive cells in target organs, and dysregulation of proinflammatory cytokines [18]. Although important differences remain between GVHD in humanized NPG mouse models within humans, key mechanisms of GVHD pathogenesis are shared in human and xenogeneic aGVHD. The pathogenesis of xeno-aGVHD shares important features with human GVHD such as TCR/co-stimulatory-mediated expansion of selected T cell clones that acquire mainly a Th1/Tc1 profile [20]. Furthermore, significantly increased human cell reconstitution and better immune responses, including immunoglobulin class switching and elevated human $\lg G$ responses, were also observed in NPG mice [21]. Thus, human allogeneic acute GVHD model established here may provide a more relevant approach for studies of human immunopathogenesis and therapeutics for aGVHD after BMT. Our data 
show that the murine inflammatory environment is capable of activating human $T$ cells to produce acute GVHD pathology regardless of whether human APCs are co-transplanted. We elected to infuse $3 \times 10^{6}$ PBMCs from donors following $200 \mathrm{cGy}$ irradiation, since we previously found that infusion of $3 \times 10^{6}$ PBMCs from donors induced a moderate GVHD in that model while administration of $7 \times 10^{6}$ to $9 \times 10^{6}$ PBMCs resulted in very severe GVHD (data not publish). In addition, irradiation dose is proportional to the degree of tissue damage and the subsequent cytokine storm, and thus is directly proportional to aGVHDrelated mortality in the mouse [22,23], our results show that low dose irradiation before PBMCs translation is necessary cause mouse antigen exposure and succeed induce x-aGVHD model.

hAMSCs are a novel source of stem cells that can be obtained in large quantities. Different from BMSC, MSCs isolated from placenta exhibit greater proliferative and differentiative potentials than BMSCs, most likely because of the early embryologic origin of amniotic membrane (AM-) and placenta (PL-) MSCs as compared with BMMSCs [24, 25]. hAMSCs have intermediate levels of HLA major histocompatibility complex (MHC) class I molecules, but do not have HLA class II antigens, FAS ligand, and the costimulatory molecules, therefore, do not activate alloreactive T cells $[26,27,28]$. Our results indicated that hAMSCs showed fibroblast-shaped morphology and adherence to plastic, the cell-surface markers of more than $90 \%$ of the hAMSCs were positive for CD90, CD105 and CD73 while negative for CD45, CD11b, HLA-DR and CD34. These findings met the criteria for MSCs identification by The Association of International Cell Therapy. Our observations further demonstrate GFP-labeled hAMSCs have better growth for tracers in the mouse, the labeling rate was $95 \%$. hAMSCs labeled with GFP fluorescence could be reproducibly and noninvasively detected by immunofluorescence and flow cytometry in lung, liver, spleen and gut on the first day and $3^{\text {th }}$ day respectively after cell infusion via the mouse tail vein. This phenomenon may be caused by secreted cytokines such as fibroblast growth factor, chemokine receptors and stem cell homing factors[29, 30,31]secreted by hAMSCs and rich marginal blood flow in that region, which are beneficial for cell migration. We also found that from day one to day three, GFP labeled cells increased gradually and were observed on lung but also can track to liver, spleen and gut. It has been [32] reported that BMSCs are largely trapped in the lungs, liver, and spleen with abundant capillaries after intravenous transplantation, our data were similar to this research but hAMSCs also can still reach the target organ early such as small intestine and liver. Therefore, hAMSC owns the ability of homing to the damaged tissues, relieving the severity of inflammation and accelerating tissue repair. Homing of MSCs may also be associated with local microvascular changes, increased capillary permeability, hemostasis, and passive retention.

We then analyzed the function of human amniotic mesenchymal stem cells (hAMSCs) in xeno-aGVHD by using the humanized mice. We revealed that hAMSCs therapy reduced villous blunting and lymphocyte infiltration into the lamina propria of the gut while reducing vascular endothelialitis and lymphocyte infiltration into the parenchyma of the liver and lung. In addition, hAMSCs suppresses xenogenesis $\mathrm{CD} 3+/ \mathrm{CD} 4+$ and $\mathrm{CD} 3+/ \mathrm{CD} 8+\mathrm{T}$ cell proportion and increase the frequency of Treg cells in target organs. The induction of immune tolerance involves a precise balance between activation and inhibition of $T$ cell responses, which is important in the development of aGVHD [33]. L.M. Tobin et al [34] found that BMSCs 
can block TNFa secretion by DC as promoting IL-10 and IL-4 secretion, which impedes T cell differentiation into Th1 cells, directing these cells to differentiate into Treg and Th2 cells respectively [35]. However, few reports about the related effect of hAMSC in vivo. We examined the $\mathrm{CD} 3^{+} \mathrm{CD} 4^{+}$and $\mathrm{CD}^{+}{ }^{+} \mathrm{CD} 8^{+} \mathrm{T}$ cell in peripheral blood and aGVHD target organs, and found a significant decrease in the proportion of $\mathrm{CD}^{+}{ }^{+} \mathrm{CD} 4^{+}$and $\mathrm{CD} 3^{+} \mathrm{CD} 8^{+} \mathrm{T}$ cells in those above tissues of mice treated with the hAMSCs compared to the PBS controls.

Our observations further demonstrate that the proportion of $\mathrm{CD} 4^{+} \mathrm{CD} 25^{+} \mathrm{Foxp} 3^{+}$Treg cells are increased after hAMSCs treatment in liver, spleen, lung, gut and blood. Our current data is similar with the group who shown that murine $\mathrm{CD} 4^{+} \mathrm{CD} 25^{+} \mathrm{Foxp}^{+}$Tregs were induced during GVHD after allogeneic $\mathrm{BMT}$, and the induction of these Tregs was correlated positively with the protection of GVHD in mice [36]. GVHD involves a pathophysiology that includes host tissue damage, increased secretion of proinflammatory cytokines (TNF-a, IFN- $y$, IL-1, IL-2, IL-12), and the activation of DCs and macrophages, NK cells, and cytotoxic T cells [37]. Inhibition of proinflammatory cytokines has been shown to be beneficial in resolution of the severity and incidence of $\operatorname{GVHD}[38,39]$ Although some in vitro studies suggest that IL10 or TGF- $\beta$ may be involved in the suppression of MSC [40,41], it remains unknown whether these molecules participate in the suppression mediated by hAMSCs in vivo. Here, we found that the amounts of IL-17A, IFN-y, TNF and IL-2 in target organs such as liver, lung and gut decreased after hAMSCs treatment. IL-17A was initially reported to be produced by T helper 17(Th17) cells [42].

Our data also show that hAMSC treatment can inhibit IL-17A, INF- $y$, TNF and IL-2 that involved in the pathogenesis of GVHD target organs. Th17 cells and Th17-associated cytokines play a central role in the occurrence of aGVHD $[43,44]$. IL-17 contributed to the development of aGVHD in recipient mice by recruiting or priming Th1 cells during the early stages of the disease, reflecting a shift from Th1 to Th17 cells in the physiopathology of aGVHD [43]. A subset of Th17 cells in the gut has been described as having regulatory properties with high levels of IL-10 and low TNF and IL-2 production [45]. We found that both IL-17A and TNF are increased in the gut of aGVHD humanized model, after hAMSC treatment, the level of IL-17A, TNF were significantly decreased. It has become well established from both murine studies and immune reconstitution data in the clinic that IFN-yis an adverse effect of GVHD-associated cytokine[46, 47, 48]. Sudeepta Aggarwal et al [49] suggested that MSCs can inhibit IFN-yand increase IL-4 secretion, may orchestrate a shift from the prominence of proinflammatory Th1 cells toward an increase in anti-inflammatory Th2 cells, beneficial for GVHD management. Other studies also showed that high level of IL-2 might favor the exacerbation of T cell-mediated inflammation rather than the survival of Tregs under proinflammatory conditions [50]. Furthermore, hAMSC can decreased the level of IFN-yin liver, lung, gut and blood and decreased the level of IL-2 in gut and blood simultaneously. hAMSCs possess potent immunomodulatory properties capable of suppressing allogeneic $T$ cell responses in vivo. The immune suppressive activity of hAMSCs in vivo was associated with a significant decrease in Th1 cytokines, including IFN- $y$ and TNF-a IL-17A and IL-2. 
In summary, using humanized mice with a complete human immune system, we successfully established a human allogeneic acute GVHD model. Using this model, we demonstrated that hAMSCs can control acute GVHD by regulating balance of Treg versus T effector cells. Therefore, our study provided a proof of concept of treatment using hAMSCs to control GVHD after BMT. This strategy could readily be extended to human clinical trials using hAMSCs alone or in combination with minimal conventional immunosuppression to control GVHD. Furthermore, our data also demonstrate that the pathogenesis of aGVHD shares important features with human GVHD and that NOD-Prkdc ${ }^{\text {scid } \| 2 r^{\text {null }}}$ (NPG) mice could serve as better model to study GVHD.

\section{Conclusions}

NOD-Prkdc ${ }^{\text {scid }} \mid 2$ ry ${ }^{\text {null }}$ (NPG) mice are a valuable tool for studying Graft versus-Host-Disease induced by human immune cells. hAMSCs can control acute GVHD via

decreasing inflammatory cytokine secretion within target organs through modulating balance of Treg versus $T$ effector cells in humanized mice. This strategy could be extended to human clinical trials using human hAMSC alone or incombination with minimal conventional immunosuppression to control acute GVHD.

\section{Abbreviations}

NPG: NOD-Prkdc ${ }^{\text {scid } \| 2 r y}{ }^{\text {null; }}$ SCID: Severe combined immune deficiency; Prkdc: DNA-dependent protein kinase catalytic subunit; NOD-SCID: non-obese diabetic-SCID; NK: natural killer; II2ry: Interleukin-2 receptor g common chain; NSG: NOD/SCID-IL2Ry ${ }^{\text {null }}$, NOD.Cg-Prkdc ${ }^{\text {scid }} \| 2 \mathrm{ry}^{\mathrm{tm} 1 \text { Wjl} / S z J ; ~ N O G: ~ N O D . C g-P r k d c ~}{ }^{\text {scid_ }}$

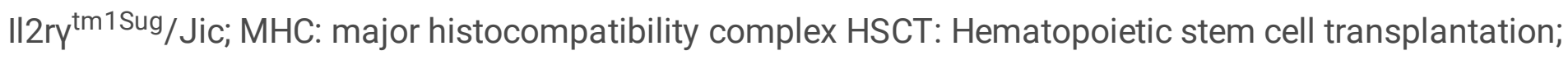
xeno-GVHD: Xenogeneic GVHD; GVHD: Graft-versus-host disease; MSCs: Mesenchymal Stem Cells; hAMSCs: human amniotic mesenchymal stem cells; BMSC: bone marrow mesenchymal stem cells; PBMC: Peripheral blood mononuclear cell

\section{Declarations}

\section{Acknowledgements}

The authors thank teachers in the Department of Laboratory Medicine, Nanfang Hospital, Southern Medical University for the excellent technical assistance.

\section{Authors' contributions}

Ping Baohong and Wang Qian conceived and designed the study, Gao Ya, Li Weiru, Bu Xiaoyin, Cai Shengchun, Zhong Jinman, Xu ying, He Yongjian and Du Meixue performed the experiments. Huang Liping collected and provided the placenta of healthy women. Gao Ya and Li Weiru wrote the paper, Jin 
Hua, Sun Haitao and Hu Xiumei reviewed and edited the manuscript. Liu Qifa participated in the discussion. All authors read and approved the final manuscript.

\section{Funding}

The authors are indebted to gratefully acknowledge Natural Science Foundation of China (81701243, 81870145,81772244), Science and Technology Planning Project of Guangzhou (201904010481), Natural Science Fund of Guangdong Province(2020A1515010038), Presidential Foundation of Nanfang Hospital of Southern Medical University (No. 2017C030), and Presidential Foundation of Zhujiang Hospital of Southern Medical University (No. yzjj2018rc03).

\section{Availability of data and materials}

The datasets used and/or analyzed during the current study are available from the corresponding author on reasonable request.

\section{Ethics approval and consent to participate}

The study was approved by the Ethics Committee of Nanfang Hospital, Southern Medical University. Signed informed consent with permissions to report individual patient data was obtained from all participants after a detailed description of the purposes of our study.

\section{Consent for publication}

Not applicable.

\section{Competing interests}

The authors declare that they have no competing interest.

\section{Author details}

*These authors contributed equally to this work

1.Department of Hematology, Nanfang Hospital, Southern Medical University, Guangzhou 510515, China

2.Department of Huiqiao, Nanfang Hospital, Southern Medical University, Guangzhou 510515, China

3.Department of Laboratory Medicine, Nanfang Hospital, Southern Medical University, Guangzhou 510515, China

4.Department of Laboratory Medicine, Zhujiang Hospital, Southern Medical University, Guangzhou, 510280 Guangdong, China

5.Department of Obstetrics and Gynecology, Nanfang Hospital, Southern Medical 
University, Guangzhou, Guangdong, China

\section{References}

1. Ghimire S, Weber D, Mavin E, et al. Pathophysiology of GvHD and Other HSCT-Related Major Complications. Front Immunol. 2017 Mar 20; 8:79.

2. Blazar BR, Murphy WJ, Abedi M. Advances in graft-versus-host disease biology and therapy. Nat Rev Immunol. 2012 May 11;12(6):443-58.

3. Wolf $D$, von Lilienfeld-Toal M, Wolf AM, et al. Novel treatment concepts for graft-versus-host disease. Blood. 2012 Jan 5;119(1):16-25.

4. Tan Y, Xiao H, Wu D, et al. Combining therapeutic antibodies using basiliximab and etanercept for severe steroid-refractory acute graft-versus-host disease: A multi-center prospective study. Oncoimmunology. 2017 Jan 6;6(3): e1277307.

5. Le Blanc K, Frassoni F, Ball L, et al; Developmental Committee of the European Group for Blood and Marrow Transplantation. Mesenchymal stem cells for treatment of steroid-resistant, severe, acute graft-versus-host disease: a phase II study. Lancet. 2008 May 10;371(9624):1579-86.

6. Insausti CL, Blanquer M, García-Hernández AM, et al. Amniotic membrane-derived stem cells: immunomodulatory properties and potential clinical application. Stem Cells Cloning. 2014 Mar 24; 7:53-63.

7. Ono M, Ohnishi S, Honda M, et al. Effects of human amnion-derived mesenchymal stromal cell transplantation in rats with radiation proctitis. Cytotherapy. 2015 Nov;17(11):1545-59.

8. Hong JQ, Gao Y, Song J, et al. Comparison of Biological Characteristics and Immunosuppressive Activity between Human Amniotic Mesenchymal Stem Cells and Human Bone Marrow Mesenchymal Stem Cells. Zhongguo Shi Yan Xue Ye Xue Za Zhi. 2016 Jun;24(3):858-64.

9. Yamahara K, Hamada A, Soma T, et al. Safety and efficacy of amnion-derived mesenchymal stem cells (AM01) in patients with steroid-refractory acute graft-versus-host disease after allogeneic haematopoietic stem cell transplantation: a study protocol for a phase I/II Japanese trial. BMJ Open. 2019 Jul 9;9(7): e026403.

10. Song J, Gao Y, Zhou WB, et al. Immunomodulatory effects of human amniotic versus bone marrowderived mesenchymal stem cells on peripheral blood T lymphocytes in vitro. Nan Fang Yi Ke Da Xue Xue Bao. 2017 Jun 20;37(6):780-785.

11. Schroeder MA, DiPersio JF. Mouse models of graft-versus-host disease: advances and limitations. Dis Model Mech. 2011 May;4(3):318-33.

12. Lucas PJ, Shearer GM, Neudorf S, et al. The human antimurine xenogeneic cytotoxic response. I. Dependence on responder antigen-presenting cells. J Immunol. 1990 Jun 15;144(12):4548-54.

13. Ito R, Takahashi T, Katano I, et al. Current advances in humanized mouse models. Cell Mol Immunol. 2012 May;9(3):208-14. 
14. Godoy JAP, Paiva RMA, Souza AM, et al. Clinical Translation of Mesenchymal Stromal Cell Therapy for Graft Versus Host Disease. Front Cell Dev Biol. 2019 Nov 21; 7:255.

15. Lim JE, Son Y. Endogenous Stem Cells in Homeostasis and Aging. Tissue Eng Regen Med. 2017 Nov 20;14(6):679-698.

16. Knuth CA, Kiernan CH, Palomares Cabeza V, et al. Isolating Pediatric Mesenchymal Stem Cells with Enhanced Expansion and Differentiation Capabilities. Tissue Eng Part C Methods. 2018 Jun;24(6):313-321.

17. Umezawa $A$, Hasegawa $A$, Inoue $M$, et al. Amnion-derived cells as a reliable resource for nextgeneration regenerative medicine. Placenta. 2019 Sep 1; 84:50-56.

18. King MA, Covassin L, Brehm MA, et al. Human peripheral blood leucocyte non-obese diabetic-severe combined immunodeficiency interleukin-2 receptor gamma chain gene mouse model of xenogeneic graft-versus-host-like disease and the role of host major histocompatibility complex. Clin Exp Immunol. 2009 Jul;157(1):104-18.

19. King M, Pearson T, Shultz LD, et al. A new Hu-PBL model for the study of human islet alloreactivity based on NOD-scid mice bearing a targeted mutation in the IL-2 receptor gamma chain gene. Clin Immunol. 2008 Mar;126(3):303-14.

20. Zeiser R, Blazar BR. Acute Graft-versus-Host Disease - Biologic Process, Prevention, and Therapy. N Engl J Med. 2017 Nov 30;377(22):2167-2179.

21. Yang X, Zhou J, He J, et al. An Immune System-Modified Rat Model for Human Stem Cell Transplantation Research. Stem Cell Reports. 2018 Aug 14;11(2):514-521.

22. Hill GR, Crawford JM, Cooke KR, et al. Total body irradiation and acute graft-versus-host disease: the role of gastrointestinal damage and inflammatory cytokines. Blood. 1997 Oct 15;90(8):3204-13.

23. Schwarte $S$, Hoffmann MW. Influence of radiation protocols on graft-vs-host disease incidence after bone-marrow transplantation in experimental models. Methods Mol Med. 2005; 109:445-58.

24. Mareschi K, Castiglia S, Sanavio F, et al. Immunoregulatory effects on T lymphocytes by human mesenchymal stromal cells isolated from bone marrow, amniotic fluid, and placenta. Exp Hematol. 2016 Feb;44(2):138-150.e1.

25. Sun $\mathrm{H}$, Hou Z, Yang $\mathrm{H}$, et al. Multiple systemic transplantations of human amniotic mesenchymal stem cells exert therapeutic effects in an ALS mouse model. Cell Tissue Res. 2014 Sep;357(3):57182.

26. Di Nicola M, Carlo-Stella C, Magni M, et al. Human bone marrow stromal cells suppress T-lymphocyte proliferation induced by cellular or nonspecific mitogenic stimuli. Blood. 2002 May 15;99(10):383843.

27. Blanco B, Herrero-Sánchez MD, Rodríguez-Serrano C, et al. Immunomodulatory effects of bone marrow versus adipose tissue-derived mesenchymal stromal cells on NK cells: implications in the transplantation setting. Eur J Haematol. 2016 Dec;97(6):528-537.

28. Huang Y, Yin Y, Gu Y, et al. Characterization and immunogenicity of bone marrow-derived mesenchymal stem cells under osteoporotic conditions. Sci China Life Sci. 2020 Mar;63(3):429-442. 
29. Keith MC, Bolli R. "String theory" of c-kit(pos) cardiac cells: a new paradigm regarding the nature of these cells that may reconcile apparently discrepant results. Circ Res. 2015 Mar 27;116(7):1216-30.

30. Eseonu OI, De Bari C. Homing of mesenchymal stem cells: mechanistic or stochastic? Implications for targeted delivery in arthritis. Rheumatology (Oxford). 2015 Feb;54(2):210-8.

31. Nitzsche F, Müller C, Lukomska B, et al. Concise Review: MSC Adhesion Cascade-Insights into Homing and Transendothelial Migration. Stem Cells. 2017 Jun;35(6):1446-1460.

32. Thompson M, Mei SHJ, Wolfe $D$, et al. Cell therapy with intravascular administration of mesenchymal stromal cells continues to appear safe: An updated systematic review and metaanalysis. EClinicalMedicine. 2020 Jan 17; 19:100249.

33. Thangavelu G, Blazar BR. Achievement of Tolerance Induction to Prevent Acute Graft-vs.-Host Disease. Front Immunol. 2019 Mar 6; 10:309.

34. Tobin LM, Healy ME, English K, et al. Human mesenchymal stem cells suppress donor CD4(+) T cell proliferation and reduce pathology in a humanized mouse model of acute graft-versus-host disease. Clin Exp Immunol. 2013 May;172(2):333-48.

35. Amorin B, Alegretti AP, Valim V, et al. Mesenchymal stem cell therapy and acute graft-versus-host disease: a review. Hum Cell. 2014 Oct;27(4):137-50.

36. Koreth J, Ritz J. Tregs, HSCT, and acute GVHD: up close and personal. Blood. 2013 Sep 5;122(10):1690-1.

37. Dong W, Wu X, Ma S, et al. The Mechanism of Anti-PD-L1 Antibody Efficacy against PD-L1-Negative Tumors Identifies NK Cells Expressing PD-L1 as a Cytolytic Effector. Cancer Discov. 2019 Oct;9(10):1422-1437.

38. Bruner RJ, Farag SS. Monoclonal antibodies for the prevention and treatment of graft-versus-host disease. Semin Oncol. 2003 Aug;30(4):509-19.

39. Choi SW, Reddy P. Current and emerging strategies for the prevention of graft-versus-host disease. Nat Rev Clin Oncol. 2014 Sep;11(9):536-47.

40. Ng J, Hynes K, White G, et al. Immunomodulatory Properties of Induced Pluripotent Stem Cell-Derived Mesenchymal Cells. J Cell Biochem. 2016 Dec;117(12):2844-2853.

41. El-Sherbiny YM, El-Jawhari JJ, Moseley TA, et al. T cell immunomodulation by clinically used allogeneic human cancellous bone fragments: a potential novel immunotherapy tool. Sci Rep. 2018 Sep 10;8(1):13535.

42. Steinman L. A brief history of $T(H) 17$, the first major revision in the $T(H) 1 / T(H) 2$ hypothesis of $T$ cellmediated tissue damage. Nat Med. 2007 Feb;13(2):139-45.

43. Kim KW, Moon SJ, Park MJ, et al. Optimization of adipose tissue-derived mesenchymal stem cells by rapamycin in a murine model of acute graft-versus-host disease. Stem Cell Res Ther. 2015 Oct 23; 6:202.

44. Gartlan KH, Markey KA, Varelias A, et al. Tc17 cells are a proinflammatory, plastic lineage of pathogenic CD8+ T cells that induce GVHD without antileukemic effects. Blood. 2015 Sep 
24;126(13):1609-20.

45. Esplugues E, Huber S, Gagliani N, et al. Control of TH17 cells occurs in the small intestine. Nature. 2011 Jul 17;475(7357):514-8.

46. Capitini $\mathrm{CM}$, Herby S, Milliron M, et al. Bone marrow deficient in IFN-\{gamma\} signaling selectively reverses GVHD-associated immunosuppression and enhances a tumor-specific GVT effect. Blood. 2009 May 14;113(20):5002-9.

47. Merli P, Caruana I, De Vito R, et al. Role of interferon-y in immune-mediated graft failure after allogeneic hematopoietic stem cell transplantation. Haematologica. 2019 Nov;104(11):2314-2323.

48. Alvarado LJ, Huntsman HD, Cheng $\mathrm{H}$, et al. Eltrombopag maintains human hematopoietic stem and progenitor cells under inflammatory conditions mediated by IFN- $\gamma$. Blood. 2019 May 9;133(19):20432055.

49. Aggarwal S, Pittenger MF. Human mesenchymal stem cells modulate allogeneic immune cell responses. Blood. 2005 Feb 15;105(4):1815-22.

50. Ratnasothy K, Jacob J, Tung S, et al. IL-2 therapy preferentially expands adoptively transferred donor-specific Tregs improving skin allograft survival. Am J Transplant. 2019 Jul;19(7):2092-2100.

\section{Table}

Table 1. Xeno-aGVHD clinical scoring system

\begin{tabular}{ccccccc}
\hline Score & Weight loss & Posture & Mobility & Fur & Skin & Gastrointestinal symptoms \\
\hline 0 & $<10 \%$ & normal & normal & normal & normal & normal \\
1 & $10-25 \%$ & $\begin{array}{c}\text { hunched } \\
\text { posture }\end{array}$ & $\begin{array}{c}\text { reduced } \\
\text { mobility }\end{array}$ & $\begin{array}{c}\text { ruffled } \\
\text { fur }\end{array}$ & $\begin{array}{c}\text { ulcers or scaling on } \\
\text { ears, tail, and paws }\end{array}$ & diarrhea (one day) \\
& & $\begin{array}{c}\text { severe } \\
\text { hunched } \\
\text { posture }\end{array}$ & $\begin{array}{c}\text { less or no } \\
\text { mobility }\end{array}$ & $\begin{array}{c}\text { severe } \\
\text { ruffled } \\
\text { fur }\end{array}$ & $\begin{array}{c}\text { skin lesions with } \\
\text { alopecia }\end{array}$ & diarrhea ( $>3$ days) or \\
hematochezia
\end{tabular}

\section{Figures}



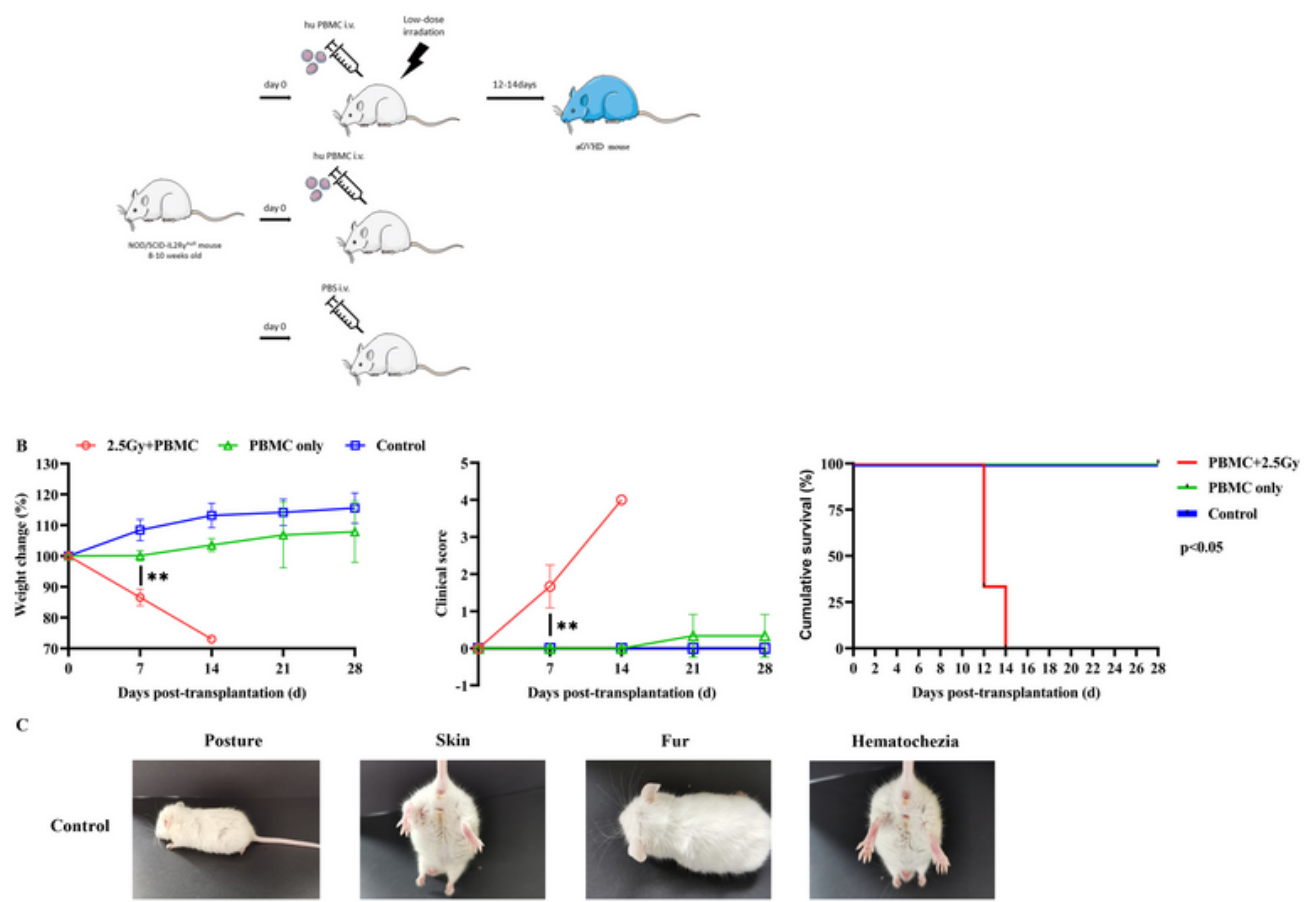

Fur
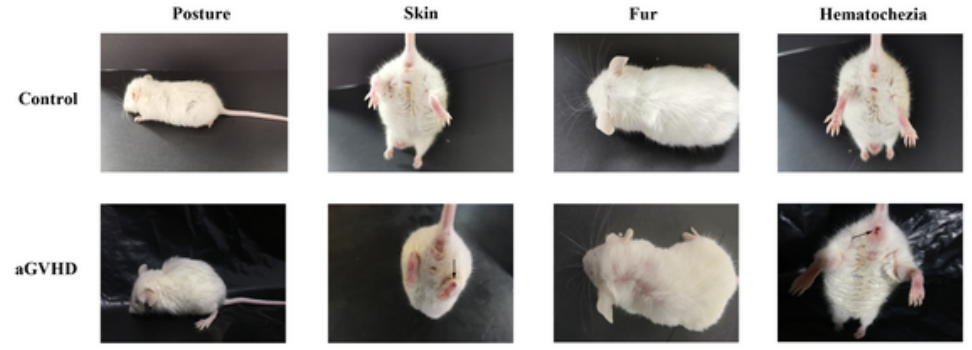

D

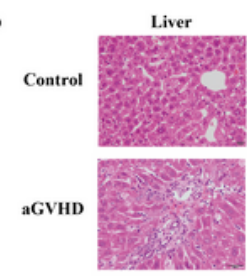

Spleen
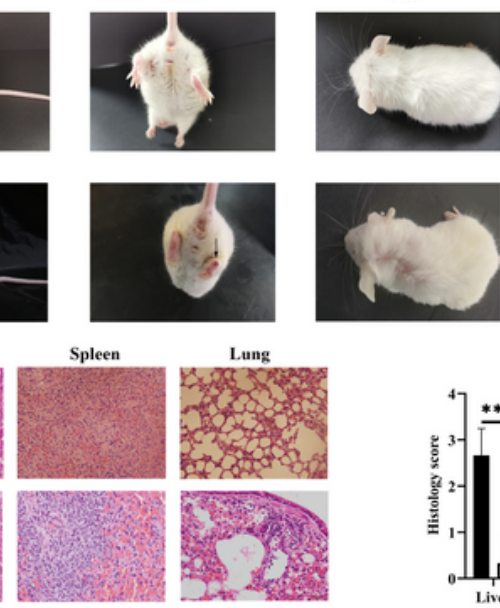

Lung
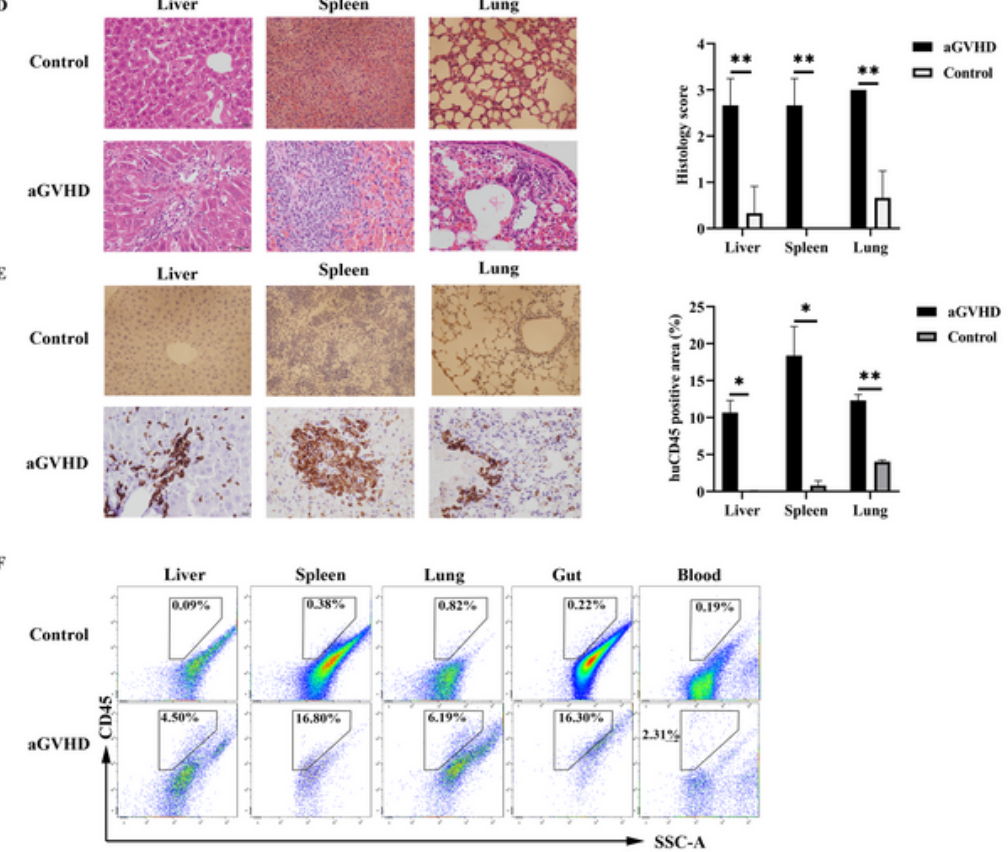

Figure 1

Low-dose irradiation before PBMC injection induced xeno-aGVHD in NPG mice. (A)Protocol for establishment of xeno-aGVHD model. NPG mouse injected with allogeneic hPBMCs or PBS after irradiated sub lethally. (B) Measurement of weight change, clinical score and survival at different time points treated with PBS, PBMC or 200 cGy followed by PBMC. The result showed that low-dose irradiation pre-conditioned accelerated the induction of xeno-aGVHD. (C) Representative clinical manifestation 
including hunched posture, ruffed fur, damaged skin and hematochezia in xeno-aGVHD mouse model. (D) Representative histology of target organs, including liver, spleen and lung, showed that inflammatory cell infiltration and tissues damaged were observed in low-dose irradiation pre-conditioned NPG mice group. 400x. (E) Representative immunohistochemistry of target organs. inflammatory cell infiltration and tissue damage in target organs such as lung, liver and spleen, human CD45+ cells were also detected by immunohistochemistry in these target organs. 400x. (F) human CD3+ T cells from each target organ were detected by flow cytometry after 3 days human PBMCs injected in vivo.

A

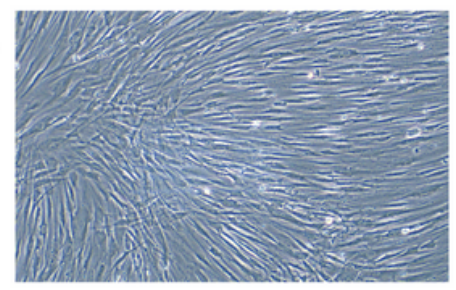

$\mathrm{C}$

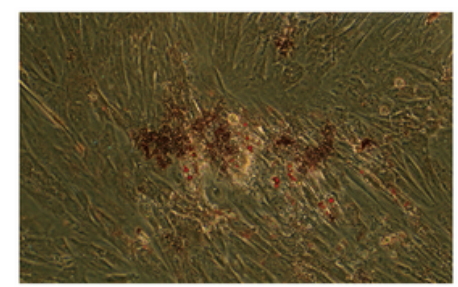

B

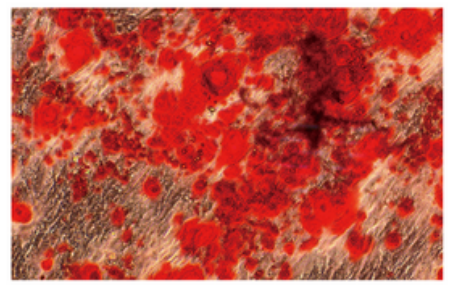

$\mathrm{E}$

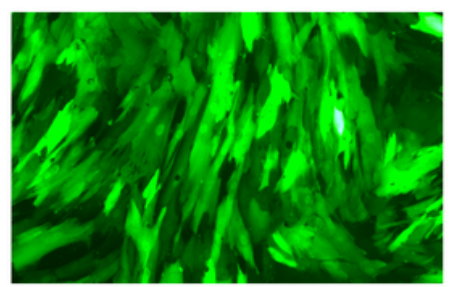

$\mathrm{D}$

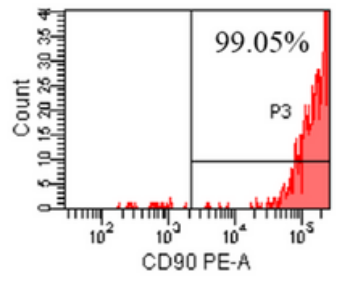

$\mathrm{CD} 90+$

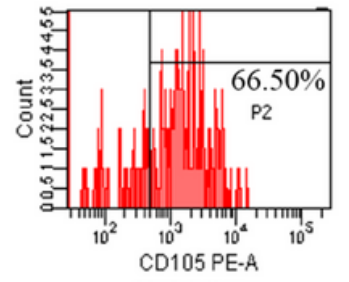

CD105+

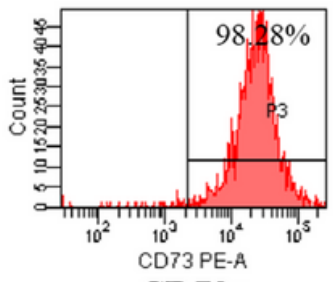

$\mathrm{CD} 73+$

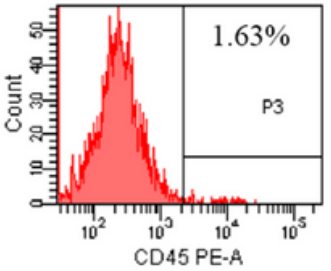

CD45-

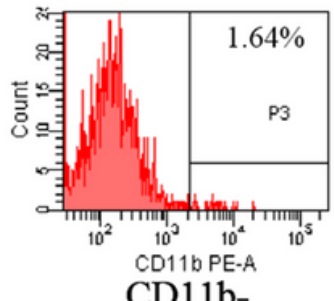

CD11b-

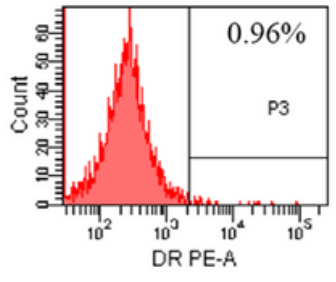

HLA-DR-

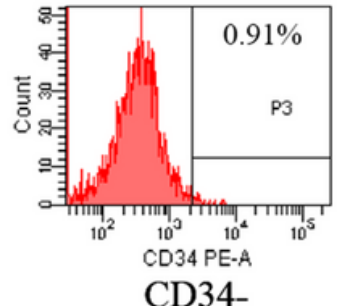

CD34-

\section{Figure 2}

Identification of human amnion mesenchymal stem cells. (A) Characteristics of passage 3 hAMSCs. (B) Osteogenic differentiation of hAMSCs which indicated by alizarin red S. 100x. (C) Adipogenic differentiation of hAMSCs, that indicated by oil red 0 staining. 100x. (D) Surface antigen of hAMSCs were detected by flow cytometry. The result showed that cells were positive for CD90, CD105 and CD73 while negative for CD45, CD11b, CD34 and HLA-DR. (E) GFP labeled hAMSCs can be detected by fluorescence microscope after 3 days transfected with GFP-pseudovirion. 100x. 
A

24h
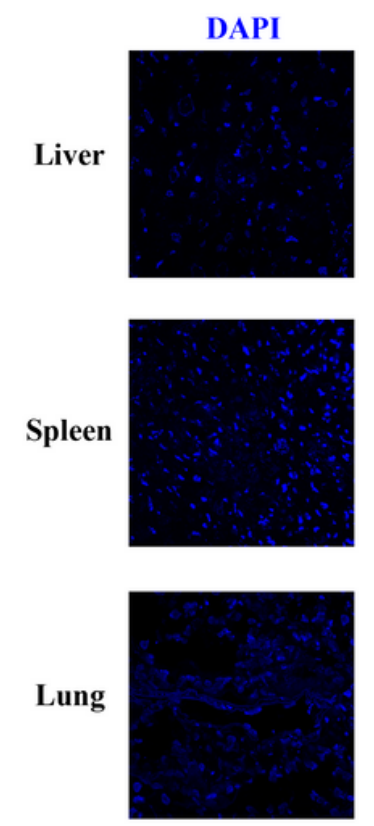

Gut

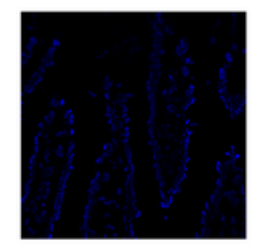

B
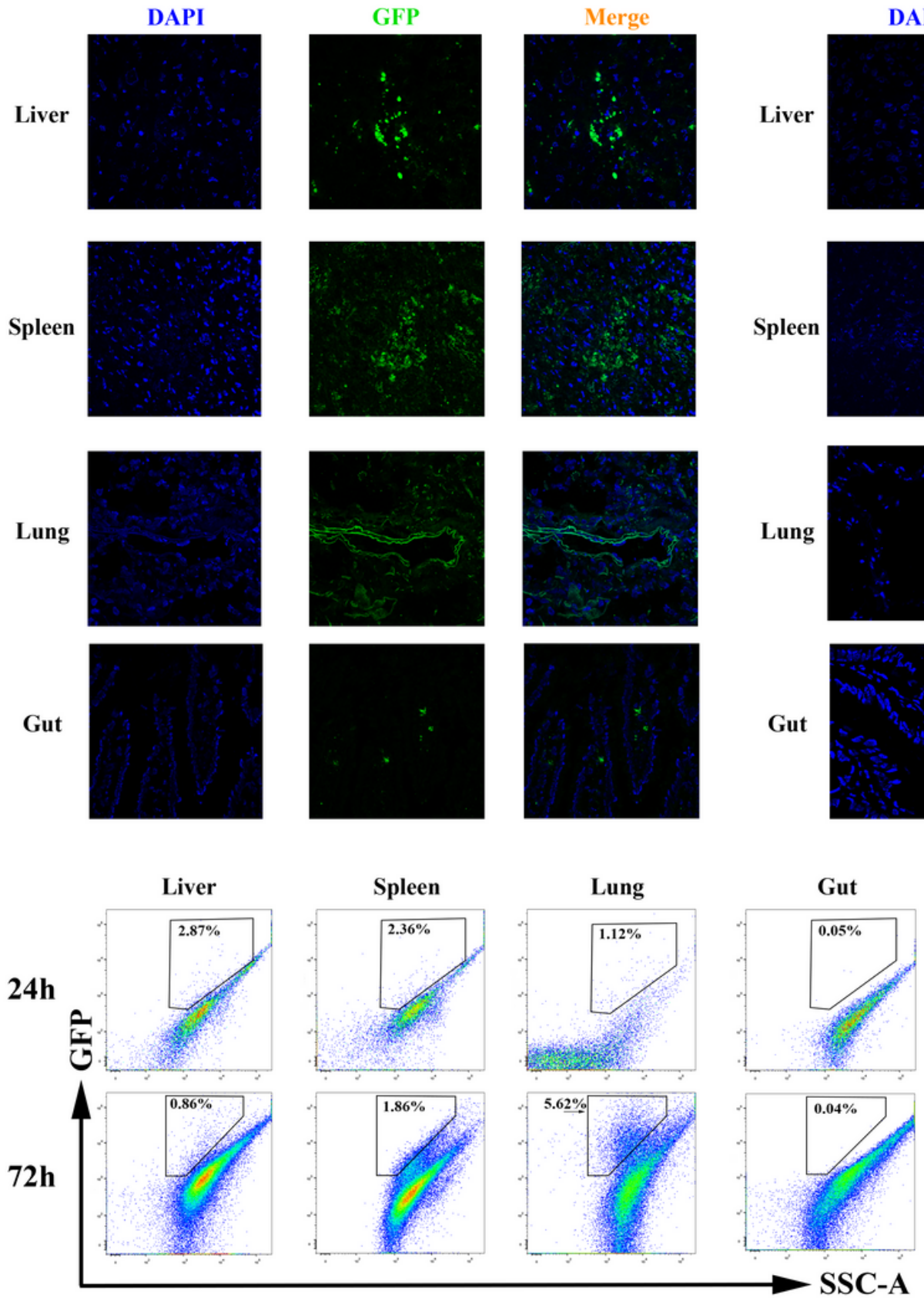

72h
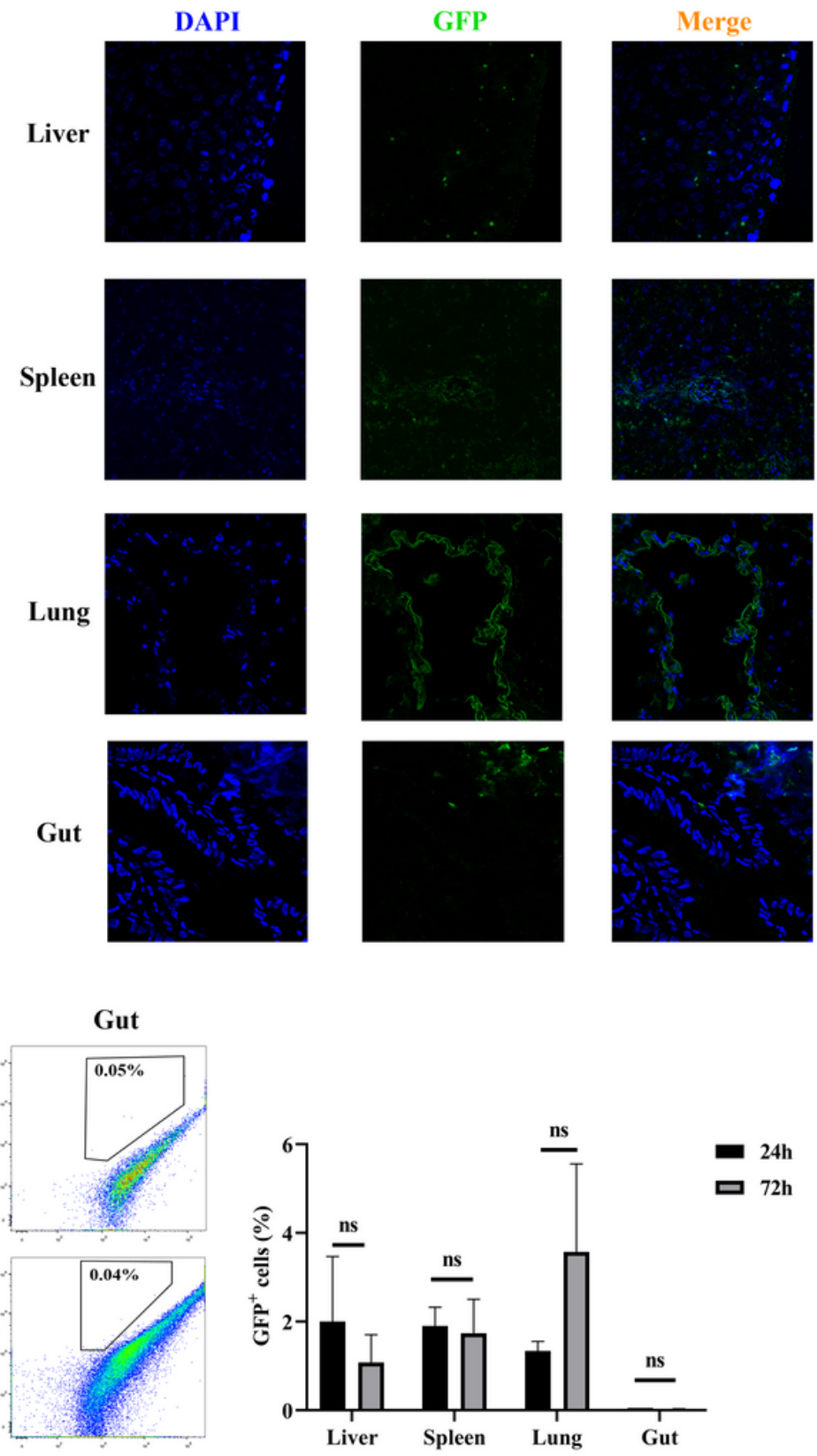

\section{Figure 3}

Human amnion mesenchymal stem cell migrated to target organs. (A) GFP labeled hAMSCs detected by confocal microscope in target organs after 24 hours injected in vivo, after 72 hours, the GFP fluorescence signal in the spleen, liver, and gut is significantly weakened while increased in lung. (B) Target organs were analyzed by flow cytometry after grinding, GFP signal could be detected in 24 hours and 72 hours respectively. 

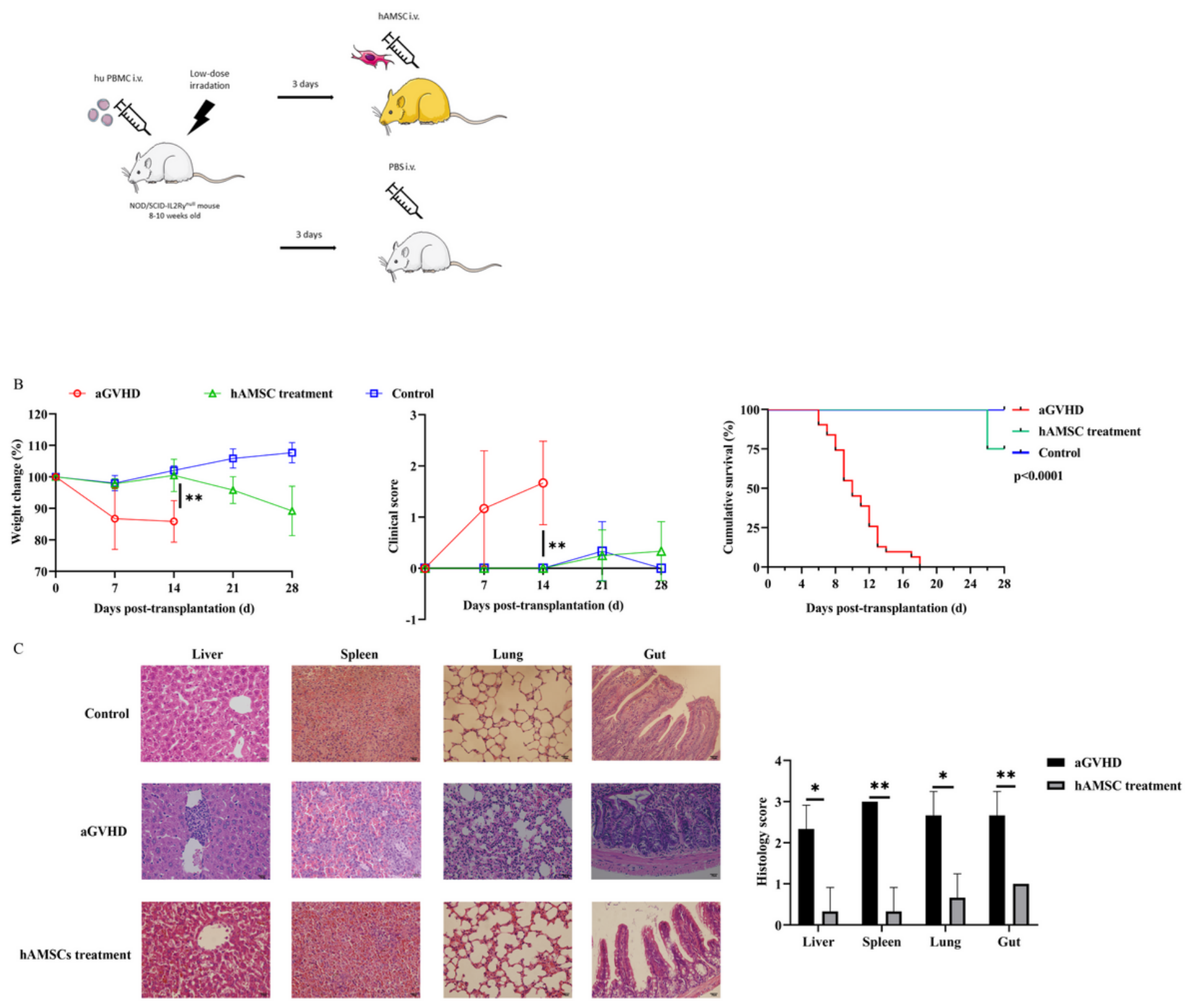

\section{Figure 4}

hAMSCs significantly inhibit the development of xeno-aGVHD. (A) Protocol for figuring out the immunosuppressed ability of hAMSCs in vivo. PBS or allogeneic hPBMCs were injected in mouse after 3 days establishing xeno-aGVHD model. (B) Measurement of weight change, clinical score and survival at different time points treated with PBS, PBMC or PBMC followed by hAMSCs. Results showed that hAMSCs can slow down the development of aGVHD. (C) Representative histology of target organs, including liver, spleen, lung and gut. Compared with hAMSCs treatment group, the inflammatory cell infiltration and tissues damaged of aGVHD group were more severe. 400x. $\left({ }^{*}, p<0.05, * \star, p<0.01, * \star *\right.$, $\mathrm{p}<0.001)$ 
A

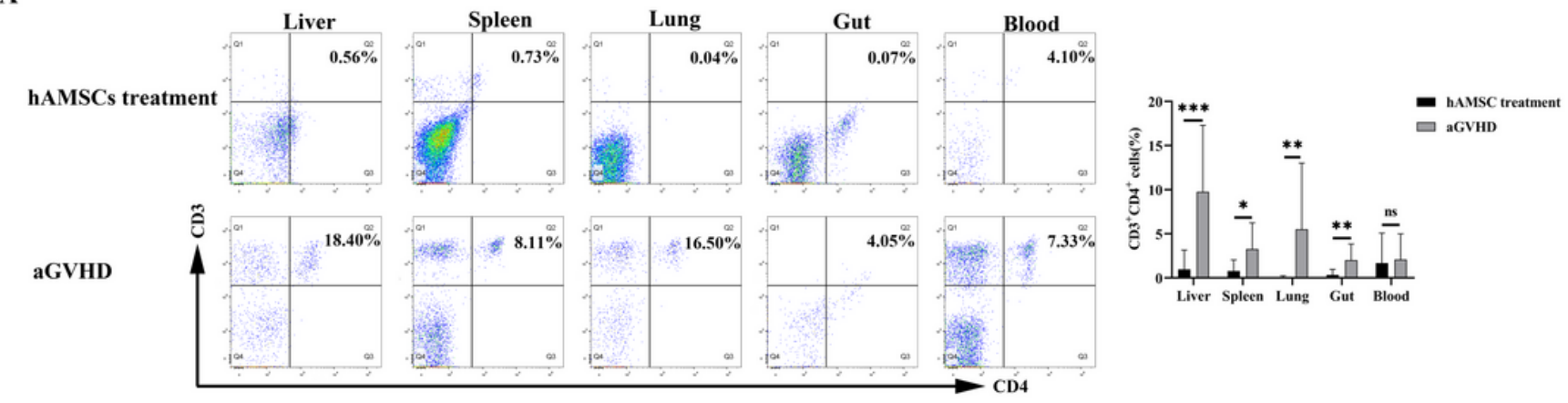

B

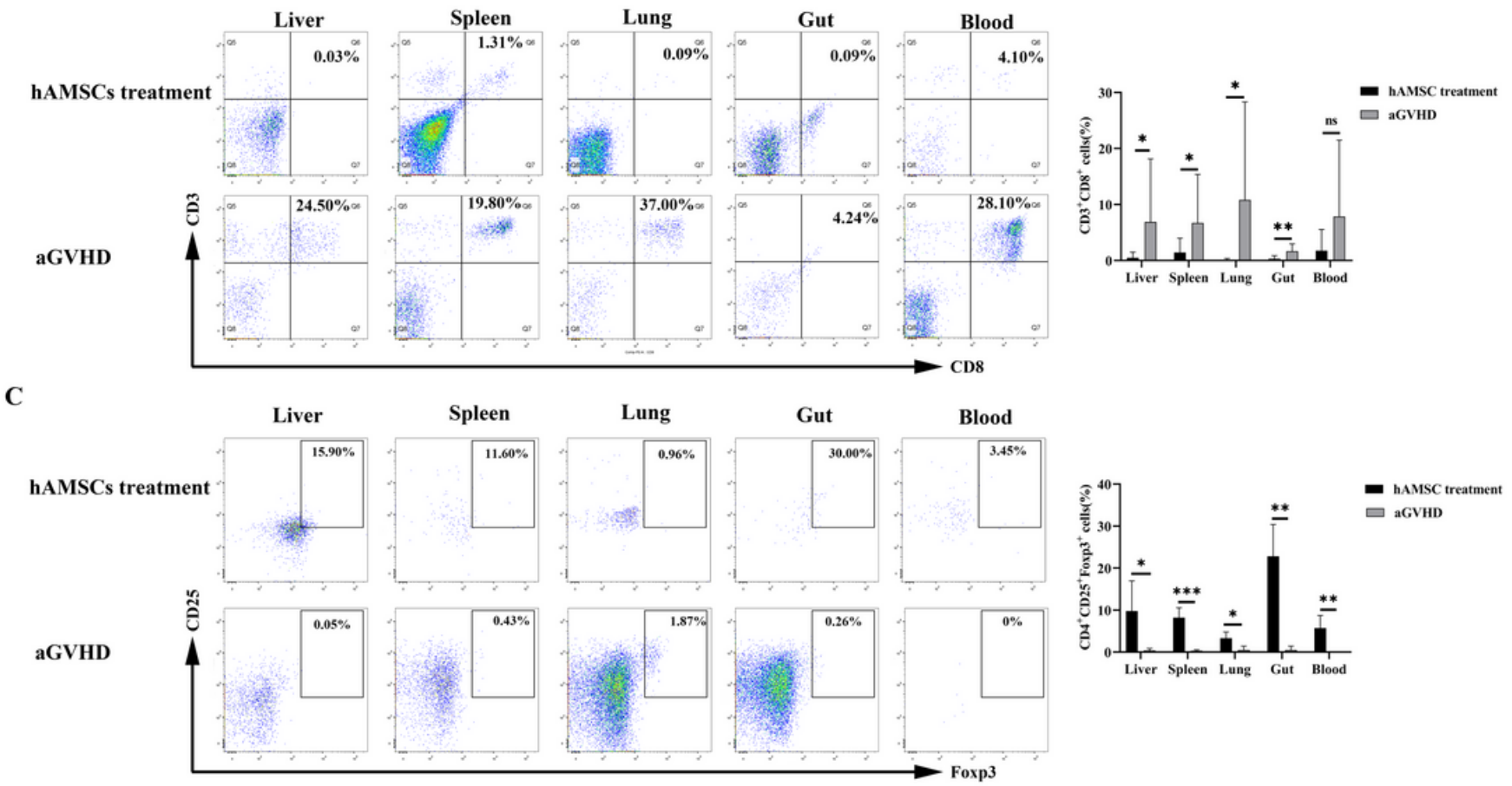

\section{Figure 5}

hAMSC suppresses CD3+CD4+ and CD3+CD8+ T cell and increases the proportion of CD4+CD25+FOXP3+Treg cells (A) T cells from each target organ were collected and then detected by flow cytometer. The frequencies of CD3+CD4+ T cells from each organ were shown. (B) The frequencies of CD3+CD8+ T cells from each organ were shown. (C) Gated on CD4, the frequencies of Tregs from each target organ were shown. $(*, p<0.05, * \star, p<0.01, * \star *, p<0.001)$ 
A

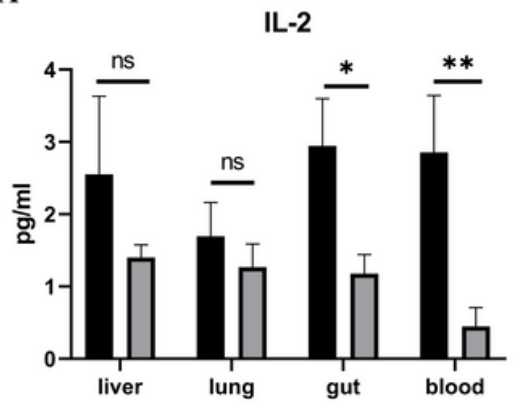

C

IL-17A

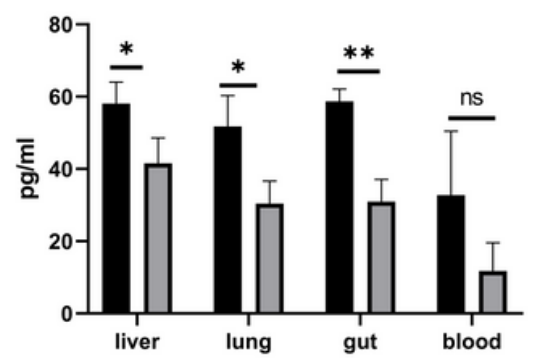

B

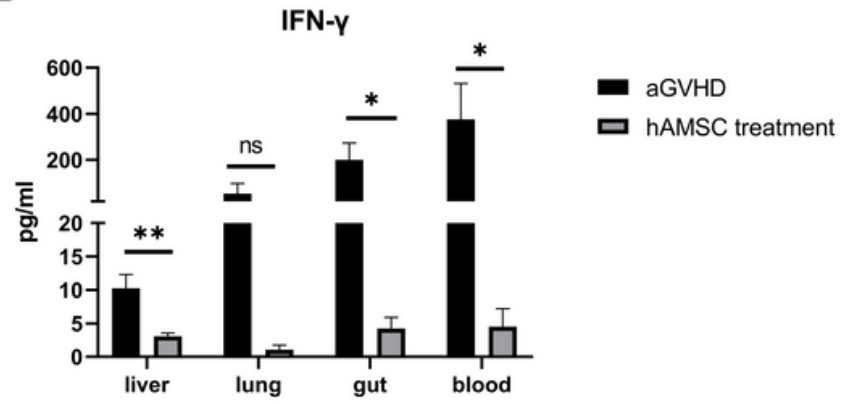

D

TNF

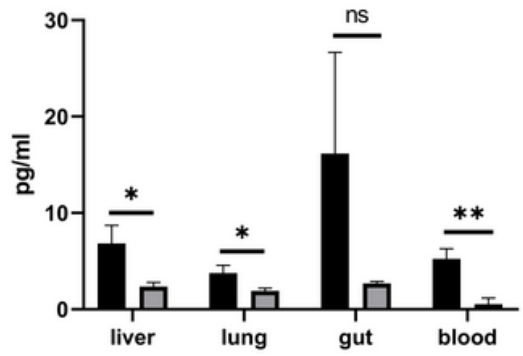

- aGVHD

口 hAMSC treatment

Figure 6

hAMSC inhibits cytosines secretion in target organs after hPBMC transplantation Concentration of cytokines (A-D) in livers, lungs, guts and blood on day 3 after transplantation in humanized GVHD mice with or without hAMSC treatment. Data are means \pm SEM and represent three independent experiments. $(*, p<0.05, * \star, p<0.01, * \star *, p<0.001)$ 